\title{
Addressing vaccine hesitancy and refusal in Canada
}

\author{
Eve Dubé PhD, Noni E. MacDonald MD MSc \\ CMAJ Podcasts: author interview at soundcloud.com/cmajpodcasts/150707-com
}

See CMAJ Open article, www.cmajopen.ca/content/3/3/E317

I n their CMAJ Open article, Wilson and colleagues report their analysis of vaccination exemptions for measles-containing vaccines among Ontario students. ${ }^{1}$ The data were extracted from the Ontario Immunization Records System over a 10-year period covering the 2002/03 to 2012/13 school years. Although vaccination exemptions remained relatively stable at less than $2.5 \%$ during this time, exemptions for nonmedical reasons significantly increased, while medical exemptions decreased. The latter trend is encouraging; as noted by the authors, investment in reducing false contraindications to vaccination seems to be working. However, even though rates of exemption in Ontario are below those seen in parts of the United States, ${ }^{2}$ the finding that nonmedical exemptions significantly increased over the study period provides hard evidence that Canada has a vaccine-hesitancy problem.

The World Health Organization (WHO) has recognized vaccine hesitancy as a growing global concern and has defined it as the "delay in acceptance or refusal of vaccines despite availability of vaccine services." A vaccine-hesitant person can delay, be reluctant (but still accept) or refuse one, some or all vaccines. ${ }^{3}$ Vaccine hesitancy is context-specific and varies across time, place and vaccine, and has many multidimensional determinants including confidence, complacency and convenience.

The WHO has recommended that all countries monitor and address vaccine hesitancy. ${ }^{3}$ Wilson and colleagues have shown how useful school vaccination registries can be for monitoring hesitancy. The geographic variability in vaccination exemptions that they noted suggests clusters of students who have not undergone vaccination either in whole or in part and who are therefore at risk of vaccine-preventable disease. Such an occurrence was recently seen in the measles outbreak in the Lanaudière region of Québec in 2015 (www.phac-aspc.gc.ca/mrwr -rhrr/2015/w13/index-eng.php).

Clearly, interventions to address vaccine hesitancy are needed. However, given that hesitancy determinants vary widely, it should come as no surprise that recent systematic reviews have shown that there is no strong evidence for a single specific intervention that can address all situations. ${ }^{3}$ The literature, however, does suggest some key principles for optimizing the development of strategies to tackle vaccine hesitancy. ${ }^{3}$ For example, multiple-component interventions, rather than single-component, are most effective. ${ }^{3}$

Substantial evidence exists to show that increasing people's knowledge, although important, is not sufficient to change behaviours. ${ }^{4}$ Communications from public health and health professionals need to move beyond the "knowledge deficit model" to adopt the more effective bidirectional approach; listening to those for whom the message is intended is as important as developing the message that experts want to communicate. ${ }^{5}$ These messages must be tailored to meet the receivers' needs. Studies have shown that, among the very hesitant, messaging that too strongly advocates vaccination may backfire to reinforce hesitancy rather than mitigate it. ${ }^{6}$ Dialogue, understanding and tailoring are key.

To address clusters of unimmunized populations such as those detected by Wilson and colleagues, the WHO Regional Office for Europe's Guide to Tailoring Immunization Programmes provides a theoretically driven framework and tools to identify vaccine-hesitant subgroups: diagnose their demand- and supply-side vaccination barriers and enablers, and design evidenceinformed responses to hesitancy tailored to the situation. ${ }^{7}$ This framework has been successfully used to address vaccine hesitancy among subgroups in several countries. ${ }^{8}$

\section{Ker POINTS}

- Although Canadian parents who hold strong antivaccination views are in the minority, the proportion of parents who can be categorized as vaccine-hesitant may be increasing.

- Despite considerable efforts, public health strategies have failed to effectively address vaccine hesitancy.

- A first and important step in developing effective strategies to combat vaccine hesitancy and refusal is to have a good understanding of both its causes and its contexts; interventions must be tailored to address the specific concerns of the individual.
Competing interests: None declared.

This article was solicited and has not been peer reviewed.

Correspondence to: Noni MacDonald, noni.macdonald@dal.ca

CMAJ 2016. DOI:10.1503 /cmaj.150707 
Another strategy that must be considered for the longer term is specifically educating children and youth in schools about vaccine needs and benefits, as well as about the risks of vaccine-preventable diseases. Based on evidence that educating children and youth can influence and has changed other behaviours, such a program could lead to fewer adults who are vaccine hesitant. ${ }^{9}$ In addition, pain at the time of vaccine administration must be addressed. Pain and fear of needles are common causes of hesitancy, and effective strategies for pain mitigation exist. ${ }^{10}$

The role of health professionals in addressing hesitancy is crucial because their recommendations strongly influence vaccine acceptance. ${ }^{11,12}$ They need to be supported in this role, especially those who are themselves vaccine hesitant. However, simply providing health professionals with talking points neither changes parental hesitancy nor providers' confidence in communicating with such parents. ${ }^{11}$ Both style and the recommendation matter; presumptive rather than participatory style is more effective with hesitant parents (e.g., "Your child needs to receive her vaccination today," v. "What do you want to do about immunization?"). ${ }^{12}$

In Canada, we need a means of easily sharing best practices in addressing vaccine hesitancy. Instead of working in silos, learning from other jurisdictions' successes and failures will ensure the development of more efficient and effective strategies. Such an endeavour will require collaboration and national leadership. Wilson and colleagues provide evidence that the Immunization Records Information System can be used to detect pockets of unimmunized populations, but it could also be used to monitor the effectiveness of vaccine-hesitancy interventions. Ontario has this registry because the province requires all school attendees to provide evidence of having undergone vaccination. Although mandatory vaccination requirements have been put forward to address hesitancy, ${ }^{3}$ Wilson and colleagues show that it is not necessarily the answer. The penalties and incentives for mandatory vaccination must be considered for it to be an effective strategy. Furthermore, for those parents who are strongly hesitant, a mandatory program may actually backfire, leading to more hesitancy and increased refusals.
A better understanding of the underlying dynamics of vaccine acceptance, hesitancy and refusal in Canada is fundamental to sustaining our vaccination programs' successes and prevent outbreaks of vaccine-preventable diseases. The importance of using a planning framework and of conducting a situational assessment to understand the root causes of the problem should not be underestimated. Careful and methodical diagnosis, tailoring the intervention to fit and evaluation of its impact are critical for success. There is no one-size-fits-all solution for addressing vaccine hesitancy.

\section{References}

1. Wilson SE, Seo CY, Lim GH, et al. Trends in medical and nonmedical immunization exemptions to measles-containing vaccine in Ontario: an annual cross-sectional assessment of students from school years 2002/03 to 2012/13. CMAJ Open 2015;3:E317-23.

2. Siddiqui M, Salmon DA, Omer SB. Epidemiology of vaccine hesitancy in the United States. Hum Vaccin Immunother 2013; 9:2643-8.

3. Report of the SAGE Working Group on Vaccine Hesitancy. Geneva: World Health Organization; 2014. Available: www.who. int/immunization/sage/meetings/2014/october/SAGE_working _group_revised_report_vaccine_hesitancy.pdf (accessed 2015 Aug. 19).

4. Cairns G, MacDonald L, Angus K, et al. Systematic literature review of the evidence for effective national immunisation schedule promotional communications. Stockholm: European Centre for Disease Prevention and Control; 2012.

5. Goldstein S, MacDonald NE, Guirguis S. Health communication and vaccine hesitancy. Vaccine 2015 Apr. 18 [Epub ahead of print].

6. Nyhan B, Reifler J, Richey S, et al. Effective messages in vaccine promotion: a randomized trial. Pediatrics 2014;133:e835-42.

7. The guide to tailoring immunization programmes. Geneva: World Health Organization; 2013.

8. Butler R, MacDonald NE; the SAGE Working Group on Vaccine Hesitancy. Diagnosing the determinants of vaccine hesitancy in specific subgroups: the guide to tailoring immunization programmes (TIP).Vaccine 2015;33:4176-9.

9. Laine J, Kuvaja-Kollner V, Pietila E, et al. Cost-effectiveness of population-level physical activity interventions: a systematic review. Am J Health Promot 2014;29:71-80.

10. Taddio A, McMurtry CM, Shah V et al Reducing pain during vaccine injections: clinical practice guideline. CMAJ 2015 Aug. 25 [Epub ahead of print].

11. Henrikson NB, Opel D, Grothaus L, et al. Physician communication training and parental vaccine hesitancy: a randomized trial. Pediatrics 2015;136:70.

12. Opel DJ, Robinson JD, Heritage J, et al. Characterizing providers' immunization communication practices during health supervision visits with vaccine-hesitant parents: a pilot study. Vaccine 2012;30:1269-75.

Affiliations: Institut national de santé publique du Québec, and Centre de recherche du CHU de Québec (Dubé), Université Laval, Que.; Dalhousie University and Canadian Centre for Vaccinology (MacDonald), IWK Health Centre, Halifax, NS

Contributors: Both of the authors contributed substantially to the conception of the article, drafted the manuscript and revised it critically for important intellectual content, approved the final version to be published and agree to act as guarantors of the work. 